\title{
Riscos Associados à automedicação do Cloridrato de Nafazolina e o farmacêutico como protagonista para o uso racional de medicamentos
}

\author{
Risks Associated with Naphazoline Hydrochloride self-medication and the pharmacist as the \\ protagonist for the rational use of medications \\ Riesgos asociados a la automedicación con Clorhidrato de Nafazolina y el farmacéutico como \\ protagonista del uso racional de los medicamentos
}

Recebido: 07/11/2021 | Revisado: 12/11/2021 | Aceito: 19/11/2021 | Publicado: 20/11/2021

\author{
Marina Inácia Maia de Melo Lima \\ ORCID: https://orcid.org/0000-0001-6944-306X \\ Centro Universitário Vale do Ipojuca, Brasil \\ E-mail: maririmlima@ outlook.com \\ Jullys Menezes Silva \\ ORCID: https://orcid.org/0000-0003-1125-2652 \\ Centro Universitário Vale do Ipojuca, Brasil \\ E-mail: menezesjullys@gmail.com \\ Lidiany da Paixão Siqueira \\ ORCID: https://orcid.org/0000-0002-4131-2313 \\ Centro Universitário Vale do Ipojuca, Brasil \\ E-mail:lidiany.siqueira@unifbv.edu.br
}

\begin{abstract}
Resumo
A nafazolina tem sua eficácia comprovada na redução das manifestações da obstrução nasal que tanto prejudica a qualidade de vida das pessoas, porém seus benefícios têm tido caminhos distorcidos, colocando em risco a saúde humana, principalmente quando existe o uso descontrolado e prevalente. Alguns excipientes utilizados no cloridrato de nafazolina podem causar a diminuição significativa da função pulmonar e reações de hipersensibilidade em pacientes asmáticos, além de poder agravar o quadro de rinite medicamentosa induzida por descongestionantes nasais. A facilidade em obter a nafazolina contribui para o agravamento de algumas patologias já pré-existentes e o aparecimento de efeitos adversos pelo uso incorreto, a dependência à substância, e em alguns casos pode ocasionar algumas outras patologias como a rinite medicamentosa, e a potencialização dos efeitos em contato com outros medicamentos. A falta de conhecimento, os riscos da automedicação, a venda sem prescrição médica, o baixo custo e a oferta destes descongestionantes são fatores que influenciam o consumo desordenado do medicamento, um grave problema de saúde pública. $\mathrm{O}$ atual estudo tem o objetivo de analisar os riscos na automedicação, e o uso incorreto de descongestionantes nasais que possuam Cloridrato de Nafazolina na sua composição e o farmacêutico como protagonista para o uso racional de medicamentos. Foram utilizados base de dados online aplicando coo descritores: cloridrato de nafazolina, nafazolina, derivados imidazolínicos, intoxicações por nafazolina, dependência à nafazolina, descongestionantes nasais, rinite medicamentosa. A orientação farmacêutica torna-se imprescindível para orientar o uso correto do medicamento, visto que este profissional possui o conhecimento específico sobre os medicamentos em geral independentemente de sua classe e quais benefícios e riscos ele pode oferecer a saúde afim de evitar futuras patologias além de contribuir com a política nacional do uso correto de medicamentos. O farmacêutico tem uma função importante em ajudar a sociedade, promovendo a educação em saúde quanto ao uso de medicamentos e seus principais efeitos adversos, intervindo na automedicação e minimizando a mesma.
\end{abstract}

Palavras-chave: Descongestionantes nasais; Imidazolina; Obstrução nasal.

\begin{abstract}
Naphazoline has been proven to be effective in reducing the manifestations of nasal obstruction, which affects people's quality of life so much, but its benefits have been distorted, putting human health at risk, especially when there is uncontrolled and prevalent use. Some excipients used in naphazoline hydrochloride can cause a significant decrease in lung function and hypersensitivity reactions in asthmatic patients, in addition to aggravating drug-induced rhinitis caused by nasal decongestants. The ease of obtaining nafazoline contributes to the aggravation of some preexisting pathologies and the appearance of adverse effects due to incorrect use, substance dependence, and in some cases it can cause some other pathologies such as drug-induced rhinitis, and the potentiation of effects in contact with other medications. The lack of knowledge, the risks of self-medication, sale without a prescription, the low cost and supply of these decongestants are factors that influence the disordered consumption of the medication, a serious public health problem. The current study aims to analyze the risks of self-medication, and the incorrect use of nasal
\end{abstract}


decongestants that have Naphazoline Hydrochloride in their composition and the pharmacist as the protagonist for the rational use of medicines.: naphazoline hydrochloride, naphazoline, imidazoline derivatives, naphazoline intoxications, naphazoline dependence, nasal decongestants, drug-induced rhinitis. Pharmaceutical guidance is essential to guide the correct use of the drug, as this professional has specific knowledge about drugs in general, regardless of their class and what benefits and risks they can offer to health in order to avoid future pathologies, in addition to contributing with the national policy on the correct use of medicines. The pharmacist has an important role in helping society, promoting health education about the use of medication and its main adverse effects, intervening in self-medication and minimizing it.

Keywords: Nasal descongestants; Imidazoline; Nasal obstruction.

\section{Resumen}

Se ha demostrado que la nafazolina es eficaz para reducir las manifestaciones de obstrucción nasal, que tanto afecta la calidad de vida de las personas, pero sus beneficios se han distorsionado, poniendo en riesgo la salud humana, especialmente cuando hay un uso descontrolado y prevalente. Algunos excipientes usados en el clorhidrato de nafazolina pueden causar una disminución significativa de la función pulmonar y reacciones de hipersensibilidad en pacientes asmáticos, además de agravar la rinitis inducida por fármacos causada por descongestionantes nasales. La facilidad de obtención de nafazolina contribuye al agravamiento de algunas patologías preexistentes y a la aparición de efectos adversos por uso incorrecto, dependencia de sustancias, y en algunos casos puede ocasionar algunas otras patologías como la rinitis inducida por fármacos, y la potenciación de efectos en contacto con otros medicamentos. El desconocimiento, los riesgos de la automedicación, la venta sin receta, el bajo costo y suministro de estos descongestionantes son factores que influyen en el consumo desordenado de la medicación, un grave problema de salud pública. El presente estudio tiene como objetivo analizar los riesgos de la automedicación, y el uso incorrecto de descongestionantes nasales que tienen Clorhidrato de Nafazolina en su composición y al farmacéutico como protagonista para el uso racional de los medicamentos.: Clorhidrato de nafazolina, nafazolina, derivados de imidazolina, nafazolina intoxicaciones, dependencia de nafazolina, descongestionantes nasales, rinitis inducida por fármacos. La orientación farmacéutica es fundamental para orientar el correcto uso del fármaco, ya que este profesional tiene conocimientos específicos sobre los fármacos en general, independientemente de su clase y qué beneficios y riesgos pueden ofrecer a la salud con el fin de evitar futuras patologías, además de contribuir con la política nacional sobre el uso correcto de medicamentos. El farmacéutico tiene un papel importante en la ayuda a la sociedad, promoviendo la educación sanitaria sobre el uso de la medicación y sus principales efectos adversos, interviniendo en la automedicación y minimizándola.

Palabras clave: Descongestionantes nasales; Imidazolina; Obstrucción nasal.

\section{Introdução}

Os medicamentos são substâncias que tem a finalidade de reparar ou realizar a manutenção da saúde, podendo apresentar diversas formas farmacêuticas como sólidas, semissólidas, liquidas, e formas especiais como aerossóis, adesivos transdérmicos e implantes (Cabral \& Pita, 2015). Geralmente são encontrados com facilidade em farmácias, entretanto existe alguns fatores que acabam promovendo a automedicação por muitas vezes não exigir receituário médico e consequentemente acarretando excesso de dosagem e intoxicações que podem causar sérios riscos à saúde, e em casos mais severos ser letal (Conselho Regional de Farmácia do Estado de São Paulo [CRFSP], 2012).

A OMS estima que, no mundo, mais da metade de todos os medicamentos são prescritos, dispensados ou vendidos inapropriadamente, e que metade dos pacientes não os usa corretamente, portanto o Conselho Federal de Farmácia inseriu, nos seus regulamentos, a prática do Uso Racional de Medicamentos que é definido como um processo que compreende a prescrição apropriada, a disponibilidade oportuna e preços acessíveis, a dispensação em condições adequadas, e o conjunto de doses indicadas nos intervalos definidos e no período de tempo indicado de medicamentos eficazes, seguros e de qualidade (João, 2010).

Apesar da prática do Uso Racional de Medicamentos, a automedicação no Brasil ainda é um problema de saúde pública que atinge a maioria dos brasileiros que utilizam medicamentos sem orientação médica ou farmacêutica, manifestando alguns riscos como intoxicações, resistências a micro-organismos, interações medicamentosas, superdosagem, e dependência de algumas substâncias (Biblioteca Virtual em Saúde do Ministério da saúde, 2012). 
A automedicação em casos de obstrução nasal é uma prática muito corriqueira, que pode ser provocada por alguma inflamação, ou irritações, infecções, gripes, resfriados, alergias, e algumas doenças crônicas como asma, rinite e sinusite, que acomete a maioria dos brasileiros (FO, 2004).

Os descongestionantes nasais possuem contraindicações, e alguns efeitos adversos podem ser desencadeados a longo prazo como arritmias cardíacas, cefaleias, insônias, irritação nasal, espirros, agitação, taquicardia, tremores e até mesmo retenção urinária (Borges et al., 2019).

Os descongestionantes nasais também podem levar á rinite medicamentosa que é provocada pelo uso inadequado de medicamentos que agridem a mucosa nasal ou agravam ainda mais a lesões já existentes, o uso prolongado desses agentes tópicos é após três dias consecutivos, causando alguns sintomas de congestão nasal iniciando com espirros constantes, isso acontece devido ao efeito rebote (Binow, 2015).

O cloridrato de nafazolina é encontrado na maioria das farmácias, como descongestionante nasal e a baixo custo. A sua ação descongestionante inicia-se em torno de cinco a dez minutos, e a duração do efeito terapêutico da nafazolina varia de três a seis horas (Bucaretchi et al., 2003).

Os derivados imidazólicos como a nafazolina são os que podem causar maiores problemas de efeito rebote, pois seu efeito nos vasos sanguíneos da mucosa nasal é longo com duração de 7 a 9 horas. O risco é maior porque a maioria dos pacientes usa a droga por períodos longos de até 90 dias ou mais de um ano em alguns casos considerados crônicos (Lague et al., 2013).

O envolvimento do farmacêutico na promoção do uso racional de medicamentos é um fator muito relevante, devido aos efeitos colaterais que o Cloridrato de Nafazolina pode causar com o uso irracional. O farmacêutico conscientizará o uso adequado, orientando na dispensação os efeitos colaterais causados pelo uso incorreto, prevenindo a automedicação posteriormente, e evitando possivelmente a dependência da substância (CRFSP, 2012).

Nesse contexto, o presente artigo objetiva analisar os riscos da automedicação, e o uso incorreto de descongestionantes nasais que possuam à Nafazolina na sua composição, ressaltando a importância do farmacêutico diante deste problema da automedicação do medicamento Cloridrato de Nafozolina e como ele pode orientar, alertar, e levar conhecimento a população ajudando a melhorar a qualidade de vida do usuário.

\section{Metodologia}

Para o presente estudo, foi realizado um levantamento bibliográfico visando uma atualização sobre Riscos associados ao uso do Cloridrato de Nafazolina o farmacêutico como protagonista para o uso racional de medicamentos. Foram efetuadas consultas nas bases de dados SCIELO, Sciense Direct e EBSCO, além dos sites oficiais como a ANVISA, Conselho Federal de Farmácia e Ministério da Saúde. Foram utilizados como descritores: cloridrato de nafazolina, nafazolina, derivados imidazolínicos, intoxicações por nafazolina, dependência à nafazolina, descongestionantes nasais, rinite medicamentosa.

Foram considerados como base dessa revisão de literatura, materiais publicados entre os anos 2003 à 2021, consistindo em ser uma revisão do tipo integrativa, com a finalidade de sintetizar os resultados obtidos em pesquisas sobre o tema de modo abrangente, oferecendo informações mais amplas sobre a problemática.

Os critérios de inclusão foram estudos que apresentassem (I) o mecanismo de ação do Cloridrato de Nafazolina e suas indicações; (II) os efeitos adversos e suas intoxicações; (III) as contraindicações e interações medicamentosas e (IV) os riscos da automedicação a longo prazo e possível dependência a substância.

Para exclusão, os critérios foram estudos que não demonstraram compreensão sobre os riscos da automedicação do Cloridrato de Nafazolina. 
Quadro 1. Artigos selecionados para análise: ano de publicação, autores e título.

\begin{tabular}{|c|c|c|}
\hline Ano & Autor & Título \\
\hline 2012 & $\begin{array}{c}\text { Biblioteca Virtual em Saúde do Ministério da } \\
\text { saúde. }\end{array}$ & Automedicação. \\
\hline 2015 & Binow, G. B. & $\begin{array}{l}\text { Rinite medicamentosa causada pelo uso abusivo } \\
\text { de descongestionantes nasais. }\end{array}$ \\
\hline 2019 & $\begin{array}{c}\text { Borges, A. S. S., Carvalho, C. G., \& Magalhães, } \\
\text { S. R. }\end{array}$ & $\begin{array}{c}\text { Riscos associados ao uso irracional do } \\
\text { descongestionante nasal: cloridrato de nafazolina. }\end{array}$ \\
\hline 2003 & Bucaretchi, F., Dragosavac, S., \& Vieira, R. J. & $\begin{array}{c}\text { Exposição aguda a derivados imidazolínicos em } \\
\text { crianças. }\end{array}$ \\
\hline 2015 & Cabral, C., \& Pita, J. R. & $\begin{array}{l}\text { Ciclo de Exposiçóes: Temas de Saúde, Farmácia } \\
\text { e Sociedade. Coimbra: Faculdade de Farmácia da } \\
\text { Universidade de Coimbra. }\end{array}$ \\
\hline 2016 & Castro, L. N., Mello, M. M., \& Fernandes, W. S. & $\begin{array}{c}\text { Avaliação da prática de automedicação com } \\
\text { descongestionantes nasais por estudantes da área } \\
\text { da saúde. }\end{array}$ \\
\hline 2012 & $\begin{array}{l}\text { Conselho Regional de Farmácia do Estado de São } \\
\text { Paulo. }\end{array}$ & $\begin{array}{l}\text { Dispensação de medicamentos. Farmácia não é } \\
\text { um comercio simples. }\end{array}$ \\
\hline 2015 & $\begin{array}{l}\text { Conselho Regional de Farmácia do Estado de São } \\
\text { Paulo. }\end{array}$ & $\begin{array}{l}\text { Descongestionantes nasais. Revista do } \\
\text { farmacêutico. }\end{array}$ \\
\hline 2020 & Dionizio, I., Macedo, L. D., \& Albino, M. A. & $\begin{array}{l}\text { A dependência de descongestionantes nasais e } \\
\text { seus efeitos colaterais. }\end{array}$ \\
\hline 2014 & Freitas, P. S. & $\begin{array}{c}\text { Eventos adversos relacionados ao uso de } \\
\text { medicamentos descongestionantes nasais tópicos }\end{array}$ \\
\hline 2004 & Fundação Otorrinolaringologista. & Obstrução nasal. \\
\hline 2010 & João, W. S. J. & Reflexões sobre o uso racional de medicamentos. \\
\hline 2021 & Júnior, D., \& Almeida, M. & $\begin{array}{c}\text { Análise do uso de descongestionantes nasais } \\
\text { isentos de corticóides no Município de Uiraúna- } \\
\text { PB. }\end{array}$ \\
\hline 2013 & $\begin{array}{c}\text { Lague, L. G., Roithmann, R., \& Augusto, T. A. } \\
\text { M. }\end{array}$ & $\begin{array}{c}\text { Prevalência do uso de vasoconstritores nasais em } \\
\text { acadêmicos de uma universidade privada do Rio } \\
\text { Grande do Sul. Revista da AMRIGS. }\end{array}$ \\
\hline 2013 & $\begin{array}{l}\text { Mello Júnior, J. F., Mion, O. G., Andrade, N. A., } \\
\text { Anselmo-Lima, W. T. A., Stamm, E. C., Almeida, } \\
\text { W. L. C., Filho, P. O. C., Castro, J. C., Padua, F. } \\
\text { G. M., Romano, F. R., Santos, R. P., Roitmann, } \\
\text { R., Voegels, R. L., Meirelles, R. C., Sá, L. C. B., } \\
\text { Moacyr, M. T., Santos, M. C. J., \& Guimarães, R. } \\
\text { E. S. }\end{array}$ & $\begin{array}{l}\text { Posicionamento da Academia Brasileira de } \\
\text { Rinologia sobre terapias tópicas nasais. Brazilian } \\
\text { Journal of Otorhinolaryngology [online]. }\end{array}$ \\
\hline 2018 & Menezes, M. P. B. & $\begin{array}{c}\text { Relatório de Estágio Profissionalizante Farmácia } \\
\text { da Misericórdia de Angra do Heroísmo. }\end{array}$ \\
\hline 2005 & National Center for Biotechnology Information. & $\begin{array}{c}\text { PubChem Compound Summary for CID 4436, } \\
\text { Naphazolin. }\end{array}$ \\
\hline 2009 & $\begin{array}{c}\text { Nigro, C. E. N., Nigro, J. F. A., Mion, O., Jr, J. F. } \\
\text { M. }\end{array}$ & $\begin{array}{l}\text { Válvula nasal: anatomia e fisiologia. Revista } \\
\text { Brasileira de Otorrinolaringologia [online]. }\end{array}$ \\
\hline- & $\begin{array}{c}\text { Salgado, M. K., de Matos, J. O., Borges, G., } \\
\text { Veado, N. C. S., Della Junior, A. P., Soares, } N . \\
\text { C., ... \& Leahy, L. }\end{array}$ & $\begin{array}{c}\text { INTOXICAÇÃO POR USO CRÔNICO DE } \\
\text { NAFAZOLINA. }\end{array}$ \\
\hline 2019 & Santos, C. R. D., \& Mello, F. D. M. C. D. & $\begin{array}{l}\text { Perfil clínico-epidemiológico das exposições por } \\
\text { nafazolina em crianças registradas no Centro de } \\
\text { Informação e Assistência Toxicológica de Santa } \\
\text { Catarina. } \\
\end{array}$ \\
\hline 2014 & Santos, A.R.F.D. & $\begin{array}{c}\text { Descongestionantes nasais-perfil de } \\
\text { utilização, efeitos adversos e interações } \\
\text { medicamentosas (Doctoral dissertation). }\end{array}$ \\
\hline 2021 & $\begin{array}{l}\text { Silva, A. A., Silva, C. M., Costa, E. T., \& Borges, } \\
\text { Q. I. }\end{array}$ & $\begin{array}{l}\text { Consumo de descongestionantes nasais contendo } \\
\text { cloridrato de nafazolina em duas farmácias de } \\
\text { Poconé-MT. }\end{array}$ \\
\hline 2009 & $\begin{array}{l}\text { Wang, R., Souza, N. F. S., Fortes, J. A. R., } \\
\text { Santos, G. J., Neto, J. R. F., \& Zytinski, L. }\end{array}$ & $\begin{array}{l}\text { Síndrome do balonamento apical secundário ao } \\
\text { uso abusivo de descongestionante nasal. }\end{array}$ \\
\hline
\end{tabular}

Fonte: Dados da pesquisa (2021).

\section{Resultados e Discussão}

\subsection{Obstrução Nasal}

As Narinas são estruturas que regulam o fluxo de ar no corpo humano, no nariz as conchas nasais inferiores ao septo nasal são extremamente importantes, pois agem como válvulas regulando o fluxo de ar (Nigro et al., 2009). 
A obstrução nasal ou congestão nasal é caracterizada como uma diminuição da quantidade de ar que consegue passar pelas fossas nasais durante a inspiração, a causa mais comum de obstrução nasal é o processo inflamatório da mucosa nasal. A inflamação ocorre quando há algum tipo de estímulo do qual o tecido da mucosa precisa se defender (FO, 2004), podendo aparecer em qualquer idade, interferindo na qualidade de vida, no sono e no desempenho de atividades diárias (FO, 2004).

\subsection{Descongestionantes Nasais e Automedicação}

Os descongestionantes nasais são medicamentos utilizados para incômodos no nariz em caso de obstrução nasal e são utilizados desde o século XIX (Santos, 2014), e hoje em dia ocupam o segundo lugar entre os medicamentos mais vendidos no Brasil, por sua venda gratuita e efeito imediato, estando diretamente ligados à automedicação (Dionizio,2020).

A Automedicação significa, “a utilização de medicamentos não sujeitos a receita médica de forma responsável, sempre que se destine ao alívio e tratamento de queixas de saúde passageiras e sem gravidade, com a assistência ou aconselhamento opcional de um profissional de saúde”. (Santos, 2014).

Em 1887 o alcaloide efedrina foi a primeira destas substâncias a ser isolada, a partir de uma erva Chinesa, a Mahuang, e só anos depois Oliver e Schafer demostraram as suas propriedades vasoconstritoras. A partir desta época em anos subsequentes, outras substâncias foram sendo descobertas (Santos, 2014).

Atualmente os descongestionantes estão divididos entre as catecolaminas (efedrina, epinefrina e fenilefrina) e os imidazolínicos (nafazolina, tetraidrozilina e oximetazolina (Freitas, 2014).

Quadro 2. Principais Derivados Imidazolínicos Encontrados no Mercado Brasileiro.

\begin{tabular}{|c|c|c|}
\hline Princípio Ativo & Laboratório & Forma Farmacêutica \\
\hline \multirow[t]{9}{*}{ Nafazolina } & SANOFI MEDLEY & Solução nasal \\
\hline & FARMACÊUTICA LTDA. & \multirow[t]{2}{*}{ Solução nasal } \\
\hline & $\begin{array}{l}\text { MEDQUIMICA INDUSTRIA } \\
\text { FARMACEUTICA LTDA. }\end{array}$ & \\
\hline & $\begin{array}{l}\text { CELLERA FARMACÊUTICA } \\
\text { S.A. }\end{array}$ & \multirow[t]{2}{*}{ Solução nasal } \\
\hline & LABORATÓRIO GLOBO SA & \\
\hline & $\begin{array}{l}\text { GEOLAB INDÚSTRIA } \\
\text { FARMACÊUTICA S/A. }\end{array}$ & Solução nasal \\
\hline & Aché Laboratórios & Aerossol nasal \\
\hline & Farmacêuticos S.A & Solução nasal \\
\hline & $\begin{array}{c}\text { GERMED FARMACEUTICA } \\
\text { LTDA }\end{array}$ & Solução nasal \\
\hline \multirow[t]{2}{*}{ Oximetazolina } & EMS S/A & Solução nasal \\
\hline & $\begin{array}{c}\text { BRAINFARMA INDÚSTRIA } \\
\text { QUÍMICA E } \\
\text { FARMACÊUTICA S.A }\end{array}$ & Solução nasal \\
\hline Fenoxazolina & - & - \\
\hline Tetrizolina & - & - \\
\hline Xilemetazolina & - & - \\
\hline
\end{tabular}

Fonte: Bucaretchi, Dragosavac, and Vieira (2003) (adaptada).

\subsection{Cloridrato de Nafazolina}

O Cloridrato de Nafazolina foi introdizido ao mercado após a década de 1940 (Santos et al., 2019)., e apresenta em sua composição C14H14N2, sendo assim fracamente básica e lipofílica, com alta biodisponibilidade do trato gastrointestinal (National Center for Biotechnology Information, 2005). 
A nafazolina tem sua eficácia comprovada na redução das manifestações de obstrução e congestão nasal que tanto prejudica a qualidade de vida das pessoas, porém seus benefícios têm tido caminhos distorcidos, colocando em risco a saúde humana, principalmente quando existe o uso descontrolado e prevalente (Dionizio et al., 2020).

Segundo a ANVISA, a nafazolina é o principal derivado da imidazolina encontrada no mercado brasileiro como descongestionante, e quando se trata de automedicação, o medicamento é o mais procurado (Freitas, 2014).

\subsection{Dados Epidemiológicos}

A intoxicação por nafazolina ocorre principalmente nos meses mais frios do ano, devido ao aumento do uso como tratamento sintomático para infecções de vias aéreas superiores o qual é um medicamento de livre acesso, foi mostrado que em crianças é percebido intoxicações graves com ingestão oral de nafazolina em doses maiores do que $0,1 \mathrm{mg} / \mathrm{kg}$, que podem corresponder a quantidade entre 1 e $2 \mathrm{~mL}$ da maioria das soluções comerciais. Apesar disso, percebe-se que esta medicação é empregada com frequência, já que pode ser adquirida sem prescrição médica e com baixos preços em farmácias e drogarias (Santos et al., 2019).

De acordo com a FDA, a nafazolina é considerada categoria $\mathrm{C}$ para medicamentos, e durante a gestação não é apontados efeitos teratogênicos quando administrada em mulheres gestantes. Contudo, outras drogas simpatomiméticas têm sido associadas a más formações, mas de acordo com a FDA não se sabe se a droga pode ser excretada no leite materno (Santos et al., 2019).

\subsection{Mecanismo de Ação}

A nafazolina atua sobre os receptores agonista alfa adrenérgicos, não apresenta atividade simpatomimética seletiva, ou seja, este fármaco se liga tanto a receptores alfa quanto beta adrenérgicos, suas propriedades farmacológicas são mediadas por sua ligação e estimulação de receptores alfa2-adrenérgicos pós-sinápticos periféricos localizados na mucosa nasal e são mediadas por sua conexão, gerando vasoconstrição local e, eventualmente sistêmica (Wang et al., 2009).

Os derivados imidazolínicos também podem estimular receptores alfa2-adrenérgicos pré-sinápticos dos centros de controle cardiovascular do sistema nervoso central, determinando a inibição da atividade simpática cerebral. (Wang et al., 2009). Entretanto à ligação da nafazolina aos receptores beta-adrenérgicos, ocasiona efeitos adversos como: vasodilatação secundária ou de reflexo, taquicardia, broncodilatação e aumento da lipólise (Medley, 2015; Oliveira, 2009).

Sua estrutura química mostra o núcleo imidazol, [4,5-diidro-2-(1-naftalenilmetil)-1H-imidazol]. Este núcleo confere não somente as ações adrenérgicas, mas também colinérgicas que agem nos receptores colinérgicos, bloqueando seletivamente a atividade parassimpática, ou seja, inibindo ou bloqueando a ação da acetilcolina (Ribeiro, 2017).

\subsection{Efeitos Adversos e Intoxicações}

Os descongestionantes nasais por si só podem acarretar sérios danos à saúde como intoxicações graves, palpitações, arritmias, erupções da pele, efeito rebote, síndrome do balonamento apical, acidente vascular encefálico hemorrágico, depressão neurológica e respiratória (Borges et al., 2019).

A nafazolina pode causar alguns casos hipertensão arterial transitória, palidez e sudorese, e por ser um derivado imidazolínico pode acabar determinando a inibição da atividade simpática cerebral (Bucaretchi et al., 2003).

Alguns excipientes utilizados no cloridrato de nafazolina podem causar a diminuição significativa da função pulmonar e reações de hipersensibilidade em pacientes asmáticos, além de poder agravar o quadro de rinite medicamentosa induzida por descongestionantes nasais (Borges et al., 2019). 
Segundo Wang et al. (2009) e Freitas (2014) existe relatos de casos clínicos mais graves, como a síndrome do balonamento apical (síndrome de Takotsubo). Uma síndrome cardíaca que geralmente acomete pacientes do sexo feminino, idosas e com alterações típicas na ventriculografia esquerda, dor torácica, e sem obstrução coronariana. Esses estudos concluíram que após a retirada do descongestionante, uma paciente de 84 anos, que possuía a síndrome, atestou melhora em seu quadro clínico, revelando uma provável relação entre o uso abusivo de medicamento simpatomimético e a síndrome de Takotsubo (Wang et al., 2009 \& Freitas, 2014).

\subsection{Contraindicações e Interações Medicamentosas}

Os descongestionantes nasais apresentam contraindicações para alguns pacientes como portadores de diabetes, hipertensão arterial, hipertireoidismo, e hiperplasia prostática (Borges et al., 2019).

O cloridrato de nafazolina também pode apresentar algumas interações com outros medicamentos, portanto deve-se observar as interações medicamentosas como precaução no uso de inibidores da monoaminoxidase, antidepressivos tricíclicos, que podem levar a uma potencialização dos efeitos pressóricos da nafazolina (Silva et al., 2021).

\subsection{Dependência}

O cloridrato de Nafazolina pode causar dependência, gerando a necessidade de quantidades maiores da substância para obtenção do mesmo resultado, o uso prolongado pode levar à rinite medicamentosa (CRFSP, 2015).

A anamnese médica ou farmacêutica é imprescindível na hora da prescrição do cloridrato de nafazolina, pois é nela que vai ser evitado a contraindicação da substância, no caso de alguns pacientes, diminuir a probabilidade dos efeitos adversos se manifestarem, intoxicações e interações medicamentosas durante o tratamento com nafazolina (Menezes, 2018).

A nafazolina é eficaz e pode ser utilizada sem riscos á saúde, desde que o paciente siga as orientações médicas ou farmacêuticas, obedecendo a dose, os horários e a forma de usar (Menezes, 2018).

\subsection{Relato de Caso}

MB, masculino, 20 anos, branco, natural e precedente de Curitiba - PR, apresentou um quadro de cefaleia occipital direita, latejante, com evolução de 7 dias, de evolução progressiva, sem irradiação, sem fatores de melhor ou piora, negou febre, palpitação, déficit neurológico focal dor torácica, dispneia, perda auditiva ou outros sinais e sintomas, refere queixa de congestão nasal associada em uso crônico de Nafazolina há 4 anos, porém há 3 meses em uso de 1 frasco de Nafazolina por dia, negou hábito etílico, tabagismo e uso de drogas ilícitas (Salgado).

Ao exame físico, o paciente apresentava-se consciente, comunicativo, Glasgow 15, corado, hidratado, sem sinais neurológicos focais, sem sinais meníngeos, hipertenso ( $\mathrm{PA}=210 / 100 \mathrm{mmHg}$ ), bradicárdico ( $\mathrm{FC}=43 \mathrm{bpm}$ ), eupneico ( $\mathrm{FR}=16$ ipm), e com saturação de $\mathrm{O} 2$ de 98\%, pupilas isocóricas e fotorreagentes, à rinoscopia: desvio de septo anterior para direita em áreas 3 e 4 de Cottle, obstrutivo com esporão inferior à direita. Mucosas coradas e exames laboratoriais e de imagens dentro da normalidade (Salgado).

Foi iniciado Analgesia com Dipirona $500 \mathrm{mg} / \mathrm{ml}$, amp $2 \mathrm{ml}$ Besilato de amlodipina, $5 \mathrm{mg}$ (comprimido) Clonidina, $150 \mathrm{mcg}$ (ampola $1 \mathrm{ml}$ ), Captopril $25 \mathrm{mg}$ (comprimido), uma vez que se levantou a hipótese de intoxicação por Nafazolina, optou-se pelo uso de Naloxona 0,4 mg (ampola de $1 \mathrm{ml}$ ) e suspensão da Nafazolina tópica. Obteve-se melhora dos sintomas, sem novas queixas. Após controle dos sintomas, realizou-se reavaliação. Dados vitais do paciente foram: a saturação de $\mathrm{O} 2$ de 97\%, o normocárdico ( $\mathrm{FC}=60 \mathrm{bpm})$, a pressão arterial normal-alta $(\mathrm{PA}=130 / 80 \mathrm{mmhg}$ ), paciente recebeu alta hospitalar e foi encaminhado para o ambulatório da otorrinolaringologia. Orientou-se o desmame do uso de Nafazolina, com diluição progressiva em soro fisiológico (Salgado). 


\subsection{Estratégias para o uso racional de descongestionantes nasais - Atuação do Farmacêutico}

A atuação do farmacêutico se faz necessária nas condutas para amenizar os problemas da automedicação com alguns recursos que podem solucionar este problema favorecendo o uso racional de medicamentos, orientando e acompanhando o paciente através da assistência e atenção farmacêutica, principalmente no ato da dispensação do medicamento (Castro et al., 2016).

Um dos grandes desafios da classe farmacêutica é modificar condutas e assumir a responsabilidade com a farmacoterapia, promovendo o uso racional do medicamento, por ser considerado um problema de saúde pública, o uso irracional do medicamento traz enorme potencial de contribuição do farmacêutico, integrando-o às equipes de saúde, para assim garantir a diminuição de morbimortalidades, e colaborar com a população no acesso a serviços de qualidade (Júnior, 2021).

O farmacêutico nesse quesito, vai além de dispensar o medicamento, já que a relação contínua com o paciente é necessária para que os serviços de intervenção sejam alcançados de maneira legal e ética, fomentando resultados que assegurem a efetividade da terapia medicamentosa estabelecida. Dessa forma, seria interessante averiguar outros campos que rodeiam o doente, como por exemplo, o grau de intensidade da congestão nasal, e o que isso pode influenciar em suas atividades do cotidiano, além de averiguar todo o ambiente a fim de apurar se há interações com outros medicamentos que estão fazendo uso, e verificar possíveis novas patologias desenvolvidas ao logo do uso crônico dessa droga, para traçar estratégias de promoção e proteção da saúde dos usuários (Júnior, 2021).

\subsection{A importância do farmacêutico no Uso Racional de Medicamentos}

O farmacêutico tem uma função importante em ajudar a sociedade, promovendo a educação em saúde quanto ao uso de medicamentos e seus principais efeitos adversos, intervindo na automedicação e minimizando a mesma (Borges et al., 2019). Dessa forma o papel de educador mais uma vez se torna o único profissional em estabelecer um contato mais íntimo com o doente garantindo o uso correto de medicamentos (Júnior, 2021).

De acordo com Vidotti e Hoefler (2006), toda as pessoas que frequentam uma drogaria têm acesso ao farmacêutico, que atua como agente sanitário daquele determinado local/comunidade, cuja sua função não se limita somente à prática da dispensação, posto que deve atuar para contribuição do todo, com seu vasto conhecimento, a fim de trazer melhorias ao paciente (Júnior, 2021).

\section{Conclusão}

Apesar de não apresentar muitos estudos na literatura, a nafazolina mantém seu destaque no grupo de medicamentos mais procurados no Brasil, e consequentemente mais automedicado. No presente estudo foi demonstrados diversas situações que podem levar o uso de descongestionantes nasais que possuem a nafazolina na sua composição.

A facilidade em obter a nafazolina contribui para o agravamento de algumas patologias pré-existentes, o aparecimento de efeitos adversos pelo uso incorreto, dependência à substância, e em alguns casos pode ocasionar algumas outras patologias como a rinite medicamentosa, e a potencialização dos efeitos em contato com outros medicamentos.

A falta de conhecimento, os riscos da automedicação, a venda sem prescrição médica, o baixo custo e a oferta destes descongestionantes são fatores que influenciam o consumo desordenado do medicamento, um grave problema de saúde pública e a orientação do profissional capacitado é indispensável.

É de suma importância a orientação do farmacêutico para orientar o uso correto do medicamento, visto que este profissional possui o conhecimento específico sobre os medicamentos em geral independentemente de sua classe e quais 
benefícios e riscos ele pode oferecer a saúde a fim de evitar futuras patologias além de contribuir com política nacional do uso correto de medicamentos.

Por fim, concluímos que a importância do fortalecimento e atuação do farmacêutico faz com que planos e medidas, como a intervenção medicamentosa, e a orientação do farmacêutico ganhem mais visibilidade, atuando como fator fundamental no uso racional de medicamentos, de forma a tentar minimizar os efeitos colaterais e outros agravantes a saúde.

É extremamente importante que o uso da nafazolina seja estudado para que no futuro exista mais informações que possam auxiliar o farmacêutico na luta contra a automedicação de descongestionantes nasais que obtenham esse princípio ativo.

\section{Referências}

BVS (2012). Automedicação, 2012. Biblioteca Virtual em Saúde do Ministério da saúde. http://bvsms.saude.gov.br/bvs/dicas/255_automedicacao.html.

Binow, G. B. (2015). Rinite medicamentosa causada pelo uso abusivo de descongestionantes nasais. http://repositorio.faema.edu.br/bitstream/ 123456789/381/1/BINOW\%2c\%20G.\%20B.\%20-RINITE\%20MEDICAMENTOSA\%20CAUSADA\%20PELO\%20USO\%20ABUSIVO\%20DE\%20DESCO NGEST IONANTES\%20NASAIS.pdf.

Borges, A. S. S., Carvalho, C. G., \& Magalhães, S. R. (2019). Riscos associados ao uso irracional do descongestionante nasal: cloridrato de nafazolina. Revista Uniabeu, 12(31), 255-267. https://revista.uniabeu.edu.br/index.php/RU/article/view/3497/pdf\#.

Bucaretchi, F., Dragosavac, S., \& Vieira, R. J. (2003). Exposição aguda a derivados imidazolínicos em crianças. Jornal de Pediatria 79(6), 519-524. https://www.scielo.br/j/jped/a/xLS39xd4jvTdBBtGztgKLnP/?lang=pt. https://doi.org/10.1590/S0021-75572003000600010.

Cabral, C., \& Pita, J. R. (2015). Ciclo de Exposições: Temas de Saúde, Farmácia e Sociedade. Coimbra: Faculdade de Farmácia da Universidade de Coimbra. 36 p. https://www.uc.pt/ffuc/patrimonio_historico_farmaceutico/publicacoes/catalogosdeexposicoes/catalogo_2exp.pdf.

Castro, L. N., Mello, M. M., \& Fernandes, W. S. (2016). Avaliação da prática de automedicação com descongestionantes nasais por estudantes da área da saúde. Journal of the Heath Sciences Institute. 34(3), 163-167. https://repositorio.unip.br/ics/edicoes/2016/03_jul-set/V34_n3_2016_p163a167.pdf.

CRFSP (2012). Dispensação de medicamentos,2012. Conselho Regional de Farmácia do Estado de São Paulo. Farmácia não é um comercio simples. 1(8). http://www.crfsp.org.br/documentos/materiaistecnicos/fasciculo_8.pdf.

CRFSP (2015). Descongestionantes nasais, 2015. Conselho Regional de Farmácia do Estado de São Paulo. Revista do farmacêutico, 120 , p. 34. http://www.crfsp.org.br/revista/469-revista-120/6363-revista-do-farmaceutico-120-tecnica-e-pratica.html.

Dionizio, I., Macedo, L. D., \& Albino, M. A. (2020). A dependência de descongestionantes nasais e seus efeitos colaterais. Revista Esfera Acadêmica Saúde, 5(2), 25 - 44. https://multivix.edu.br/wp-content/uploads/2021/05/revista-esfera-saude-v05-n02-completa.pdf.

Freitas, P. S. (2014). Eventos adversos relacionados ao uso de medicamentos descongestionantes nasais tópicos - Revisão bibliográfica. Revista On-line IPOG Especialize. Goiânia, v. 8.

FO (2004). Obstrução nasal, 2004. Fundação Otorrinolaringologista. https://forl.org.br/InformacaoDoenca/Visualizar/8.

João, W. S. J. (2010). Reflexões sobre o uso racional de medicamentos. Pharmacia Brasileira, 78, 15-6. https://www.cff.org.br/sistemas/geral/revista/pdf/128/015a016_artigo_dr_walter.pdf.

Júnior, D., \& Almeida, M. (2021). Análise do uso de descongestionantes nasais isentos de corticóides no Município de Uiraúna-PB.

Lague, L. G., Roithmann, R., \& Augusto, T. A. M. (2013.). Prevalência do uso de vasoconstritores nasais em acadêmicos de uma universidade privada do Rio Grande do Sul. Revista da AMRIGS. Porto Alegre. v.57, n.1, p.39-43, Jan-mar. http://amrigs.org.br/revista/57-01/1088.pdf.

Mello Júnior, J. F., Mion, O. G., Andrade, N. A., Anselmo-Lima, W. T. A., Stamm, E. C., Almeida, W. L. C., Filho, P. O. C., Castro, J. C., Padua, F. G. M., Romano, F. R., Santos, R. P., Roitmann, R., Voegels, R. L., Meirelles, R. C., Sá, L. C. B., Moacyr, M. T., Santos, M. C. J., \& Guimarães, R. E. S. (2013). Posicionamento da Academia Brasileira de Rinologia sobre terapias tópicas nasais. Brazilian Journal of Otorhinolaryngology 79(3), 391-400. https://doi.org/10.5935/1808-8694.20130067.

Menezes, M. P. B. (2018). Relatório de Estágio Profissionalizante Farmácia da Misericórdia de Angra do Heroísmo. Faculdade de Farmácia da Universidade do Porto. Mestrado Integrado em Ciências Farmacêuticas. https://docplayer.com.br/78128401-Relatorio-de-estagio-em-farmacia-comunitaria-farmacia-damisericordia-de-angra-do-heroismo.html.

National Center for Biotechnology Information. (2005). PubChem Compound Summary for CID 4436, Naphazolin.. https://pubchem.ncbi.nlm.nih.gov/compound/Naphazoline.

Nigro, C. E. N., Nigro, J. F. A., Mion, O., Jr, J. F. M. (2009). Válvula nasal: anatomia e fisiologia. Revista Brasileira de Otorrinolaringologia. 75(2), 305-310. https://doi.org/10.1590/S0034-72992009000200024.

Salgado, M. K., de Matos, J. O., Borges, G., Veado, N. C. S., Della Junior, A. P., Soares, N. C., ... \& Leahy, L. Intoxicação Por Uso Crônico De Nafazolina. 
Research, Society and Development, v. 10, n. 15, e323101522935, 2021

(CC BY 4.0) | ISSN 2525-3409 | DOI: http://dx.doi.org/10.33448/rsd-v10i15.22935

Santos, C. R. D., \& Mello, F. D. M. C. D. (2019). Perfil clínico-epidemiológico das exposições por nafazolina em crianças registradas no Centro de Informação e Assistência Toxicológica de Santa Catarina.

Santos, A. R. F. D. (2014). Descongestionantes nasais-perfil de utilização, efeitos adversos e interações medicamentosas (Doctoral dissertation).

Silva, A. A., Silva, C. M., Costa, E. T., \& Borges, Q. I. (2021). Consumo de descongestionantes nasais contendo cloridrato de nafazolina em duas farmácias de Poconé-MT. UNIVAG - Centro Universitário de Várzea Grande. TCC-FARMÁCIA. http://www.repositoriodigital.univag.com.b r/index.php/far/article/download/660/656.

Wang, R., Souza, N. F. S., Fortes, J. A. R., Santos, G. J., Neto, J. R. F., \& Zytinski, L. (2009). Síndrome do balonamento apical secundário ao uso abusivo de descongestionante nasal. Arquivos brasileiros de cardiologia. 93(5), 75-78. https://doi.org/10.1590/S0066-782X2009001100022. 